\title{
A seven item scale for the assessment of disabilities after child and adolescent injuries
}

\author{
Rosa Gofin, Bella Adler
}

\begin{abstract}
Objectives-To develop a scale to assess physical disabilities after child or adolescent injuries.
\end{abstract}

Setting-The three main hospitals of Jerusalem.

Methods-Telephone interviews pertaining to the injury's effect on the functioning of children 4-17 years old $(n=281)$ were carried out six months after an injury. Disabilities were recalled by the parents for the period immediately after the injury (short term) and at the time of interview (long term). Of 25 questions derived from the International Classification of Impairments, Disabilities and Handicaps, seven were selected: limitations in walking, running, getting up/lying down, moving in bed, going to the toilet, bathing/keeping personal hygiene, and dressing. Construct validity was tested using the usual, sport, school, and leisure time activities as the gold standard.

Results-The prevalence of short term disabilities ranged from $23.8 \%$ to $37.7 \%$ and of long term disabilities from $0.4 \%$ to $11.8 \%$. Cronbach's $\alpha$ was 0.91 for the short term scale and over 0.90 for the different categories of the sociodemographic variables. It decreased to 0.66 for the long term scale. Sensitivity of the short term scale ranged from $77 \%$ to $89 \%$, but was lower for the long term scale. Specificity varied from $72 \%$ to $84 \%$ and increased to $88 \%$ to $90 \%$, six months after the injury.

Conclusions-This scale could be used to study disability after injury among children and adolescents in different cultures. It is a simple method that does not require expert personnel and has relatively high validity and internal reliability.

(Injury Prevention 1997; 3: 120-123)

Keywords: disabilities; validity.

Department of Social Medicine, Hadassah Medical Organization and the Braun School of Public Health and Community Medicine, Hebrew University and Hadassah, Jerusalem, Israel R Gofin B Adler

Correspondence to: Dr Rosa Gofin, Department of Socia Medicine, Hadassah Hospital, Ein Karem, POB 12000 , Jerusalem 91120 , Israel.
The magnitude of the injury problem is usually described as a 'pyramid'; at its tip is mortality, followed, in a continuously widening base for each strata, by hospitalizations, emergency room admissions, other consultations, and injuries that did not require, or for which, no attention was sought. Disabilities are seldom included in the pyramid, although most children and adolescents released from emergency rooms have limitations in activities ${ }^{12}$ as do some who experience injuries that are not medically attended. ${ }^{3}$

The assessment of disabilities is needed not only at the individual level but to determine the functional abilities of populations. At the individual level, assessment is needed fow decision on diagnosis and treatment, evalux $\frac{\bar{a}}{2}$ tion of treatment results, care needs, and need for educational and daily activities. At the population level, the assessment of the prevalence of disabilities is needed nationally regionally or locally, to evaluate health status at different points in time, and, in particular, if the case of injured children, to study outcomes other than death or recovery. Policymakets need to use this information for decision making on planning for preventive activities based, not only in the frequency and severity of injuries, but also on their impact on the child's activities. In addition, for the purpose of comparing the prevalence of disabilities afte injury across populations of different socio $\vec{\theta}$ cultural backgrounds, methods that are easy to apply, that are not culture bound, and that do not require expert personnel, are needed.

The World Health Organization (WHO International Classification of Impairments Disabilities and Handicaps (ICIDH), deve oped in the 1970s, classifies the consequencest of diseases or injury. ${ }^{4}$ According to tha classification, impairment represents the cone sequences at the level of the organ, and $\mathrm{fo}$ concerned with abnormalities of body struce ture and appearance, and with organ, system. or mental function. Disabilities represent dis turbances at the level of the person, and are concerned with the consequences of those impairments in terms of the functional perfoc mance and activity. Handicap, which reflects the interaction of the individual with his environment, is concerned with the disadvan: tages experienced as a result of the impairmen or disability.

The assessment of children's disabilities max include professional assessment, ${ }^{5}$ subjective assessment by parents, ${ }^{6}$ or by other infor mants, ${ }^{7}$ or by children themselves. ${ }^{8}$ Scales that have been developed for determining those disabilities vary in the underlying condition $\frac{N}{\omega}$ usually diseases-determining the specific oue come, in their complexity, the number of domains or items included, the target popula tion, and the type of personnel needed for their application. ${ }^{5-12}$

Most of the scales developed for children ar complex, require expert interviewers, take 8 long time to apply, and, in many cases, are culture bound. The Functional Independent Measure for children ${ }^{5}$ is applicable to childre from the ages of 6 months to 8 years. includes 18 items graded in a seven poin ordinal scale. It requires expert personne? trained for four hours, and takes about 1520 minutes to apply. The scale developed by the Rand Corporation ${ }^{6}$ for children $5-13$ years of age, includes 13 questions dealing with 
mobility, physical, self care, and role activities, and is used as a dichotomy, where the positive answer is limitation in any of the activities studied. The Pediatric Evaluation of Disability Inventory, ${ }^{11}$ designed for use in pediatric rehabilitation settings, includes 197 tasks in self care, mobility, and social function, scored as capable or not capable, with an additional 20 questions for caregiver assistance, graded on a five point scale. The scale used in Canadian studies of health and disabilities for 0-14 year olds, ${ }^{13}$ inquired about the use of technical aids, included a report on long term conditions or health problems, and used trained interviewers. Durkin et al developed a scale for use among 2-9 year olds that included 10 questions, answered as yes/no, applied by professionals, and included specific disabilities such as vision and hearing, and the presence of seizures. ${ }^{14}$ A scale developed in New Zealand for use in late adolescence included 70 questions from seven sections adopted from the ICIDH, dealing with behaviour, communications, personal care, locomotion, disposition, dexterity, and situational disability. ${ }^{15}$ Questions were asked by a trained interviewer and answered as yes/no. The most limiting disability was further examined using a $1-5$ point scale. This, in turn, was used as a screen for more detailed questioning.

Although all these measurements have been applied successfully, there remains a need for an instrument that will identify disabilities after injury among children in a wide age range, which is simple, easy to apply, and which is not culture bound.

The present study deals with the development of such a scale, for use among children and adolescents who experienced any type of injury that can be used in different cultures.

\section{Population and methods}

Details on the study population and methods are presented elsewhere. ${ }^{16}$ In brief, the study population consisted of all children, residents of Jerusalem aged $0-17$ years $(n=432)$, who were hospitalized for unintentional injuries $(\mathrm{E}$ code 800-999, $\mathrm{N}$ code $800-999)^{17}$ in three Jerusalem hospitals during one calendar year. In the present study, only children aged 4-17 were included $(n=281)$. Thirteen per cent of the children suffered intracranial injuries, $8 \%$ head fractures, $27 \%$ fractures in other parts of the body, $4 \%$ internal injuries, $8 \%$ burns, $15 \%$ open wounds, $2 \%$ sprains, and the rest had other types of injuries. The injury severity score (ISS-90) ${ }^{18}$ distribution was as follows: $30 \%$ ISS 1 and $2,44 \% 4-8$, and $26 \% 16$ or above.

Telephone interviews with the parents were carried out six months after the injury. Data on disabilities were requested for the period immediately after the injury (short term), and at the time of the interview (long term). The 25 questions asked were derived from the ICIDH ${ }^{4}$ and included personal care, locomotion, manual handling, communication, and sensitivity to pain and temperature. The answers to these questions were 'yes' or 'no'. Disabilities were defined as any limitation occurring as a consequence of the injury in the performance of any of the above mentioned 25 items of the ICIDH. Parents were also asked about limitations on daily, school, sport, and leisure time activities.

The response rate was $85 \% ; 13 \%$ of the children could not be contacted due to a wrong telephone number, no number available, or were not traced after several attempts. Only three parents refused to be interviewed. Children not included in the study did not differ in age, sex, length of hospitalization, or type and severity of injuries from those who were included.

Data were analysed by the SPSS-PC computer program. ${ }^{19}$ Reliability was assessed by Cronbach's $\alpha$. Construct validity was tested using the performance of daily, school, sport, and leisure time activities after the injury as the gold standard. For the analysis of validity, children were classified as free of disabilities if the answers were negative to all the limitations, and as having a disability if there was a positive answer to at least one of the limitations. Sensitivity was defined as the ability to identify children with disabilities (percentage of children having one or more disabilities) among those limited in activities. Specificity refers to the ability to identify disability-free children (percentage of children free of any disability) among those who were not limited in activities. Ninety five per cent confidence intervals were calculated. ${ }^{20}$

\section{Results}

Combinations of items that form the smallest subgroup out of the total of 25 items were studied to define a scale that had acceptable internal consistency-reliability and construct validity. The final selection includes seven items defining a scale that represents the range of activities performed by children (table 1). Based on these, the prevalence of short term disabilities ranged from $23.8 \%$ to $37.7 \%$. Six months after the injury, the prevalence decreased, ranging from $0.4 \%$ to $11.8 \%$.

Cronbach's $\alpha$ (table 2) was 0.91 for the short term disabilities, and over 0.90 for all but two of the categories of the sociodemographic variables listed. The exceptions, child's age group 15-17 (0.84) and mother's age group 30-34 (0.89) nevertheless had high values. For long term disabilities, Cronbach's $\alpha$ decreased to 0.66 in the total population and the values were lower than for the short term disabilities.

The scale's sensitivity and specificity was relatively high for short term disabilities

Table 1 Prevalence of disabilities ( $n=231)$; results are number (\%)

\begin{tabular}{lcc}
\hline Limitation in: & Short term & Long term \\
\hline Walking & $76(32.9)$ & $19(8.3)$ \\
Running & $84(36.4)$ & $27(11.8)$ \\
Getting up/lying down & $62(26.8)$ & $6(2.6)$ \\
Moving in bed & $55(23.8)$ & $1(0.4)$ \\
Going to toilet & $87(37.7)$ & $3(1.3)$ \\
Bathing/keeping personal hygiene & $85(36.8)$ & $4(1.7)$ \\
Dressing & $85(37.5)$ & $2(0.9)$ \\
\hline
\end{tabular}


(table 3). It identified the great majority of children with disabilities $(77.0 \%-88.8 \%)$ and those free of them $(72.4 \%-84.4 \%)$. Conversely, it missed $11.2 \%-23.0 \%$ of the disabled children, and $15.6 \%-27.6 \%$ are falsely identified as having at least one disability. Moving the cut off point to the upper tertile of the scale, which included 4-7 disabilities, decreases sensitivity (misses more children with disabilities) while improving specificity (decreases the number of children assessed as having disabilities they do not actually have). For long term disabilities, the ability to correctly identify those with disabilities was lower than for the short term scale $(52.6 \%$ $73.7 \%$ ), but the specificity was higher $(87.8 \%-90.2 \%) ; 26.3 \%-47.4 \%$ of the children with limitations of activities are not identified, and $9.8 \%-12.2 \%$ are falsely identified as positives.

Comparison with the complete 25 item scale was done using Pearson's correlation coefficient. For the short and long term scales the $r$ was high: 0.92 and 0.80 , respectively.

The disability scale was also positively

Table 2 Reliability* of the disability scale by sociodemographic characteristics

\begin{tabular}{lrll}
\hline & No & Short term & Long term \\
\hline Child's age (years) & & & \\
4-9 & 127 & 0.92 & 0.84 \\
10-14 & 63 & 0.93 & 0.44 \\
15-17 & 40 & 0.84 & 0.46 \\
Sex & 154 & 0.91 & 0.74 \\
Boys & 76 & 0.93 & 0.31 \\
Girls & & & \\
Mother's education (years) & 23 & 0.96 & 0.38 \\
0-8 & 95 & 0.91 & 0.47 \\
9-12 & 105 & 0.91 & 0.73 \\
13+ & & & \\
Mother's origin & 128 & 0.92 & 0.72 \\
Israel & 42 & 0.91 & 0.51 \\
Europe/America & 59 & 0.92 & 0.51 \\
Asia/Africa & & & \\
Mother's age (years) & 18 & 0.96 & 0.97 \\
25-29 & 43 & 0.89 & 0.57 \\
30-34 & 62 & 0.92 & 0.35 \\
35-39 & 101 & 0.92 & 0.42 \\
40+ & & & \\
Birth rank & 56 & 0.92 & 0.56 \\
1 & 56 & 0.93 & 0.79 \\
2 & 70 & 0.91 & 0.54 \\
3-4 & 46 & 0.91 & 0.30 \\
5+ & & & \\
Crowding (persons/room) & 90 & 0.93 & 0.42 \\
1.4 & 56 & 0.91 & 0.49 \\
1.5-1.9 & 86 & 0.91 & 0.83 \\
>2.0 & 230 & 0.91 & 0.66 \\
Total & & & \\
\hline
\end{tabular}

${ }^{\star}$ Cronbach's $\alpha$

Table 3 Sensitivity and specificity of the disability scale

\begin{tabular}{|c|c|c|c|c|}
\hline \multirow[b]{2}{*}{ Activities } & \multicolumn{2}{|c|}{ Sensitivity* } & \multicolumn{2}{|c|}{ Specificityt } \\
\hline & $\overline{\%}$ & $95 \% C I$ & $\%$ & $95 \% C I$ \\
\hline \multicolumn{5}{|l|}{ Daily } \\
\hline $\begin{array}{l}\text { Short term } \\
\text { Long term }\end{array}$ & $\begin{array}{l}81.1 \\
52.6\end{array}$ & $\begin{array}{l}73.3 \text { to } 87.3 \\
28.8 \text { to } 75.6\end{array}$ & $\begin{array}{l}84.4 \\
88.0\end{array}$ & $\begin{array}{l}75.5 \text { to } 91.0 \\
82.7 \text { to } 92.1\end{array}$ \\
\hline School & & & & \\
\hline $\begin{array}{l}\text { Short term } \\
\text { Long term }\end{array}$ & $\begin{array}{l}77.0 \\
73.7\end{array}$ & $\begin{array}{l}69.1 \text { to } 83.7 \\
48.8 \text { to } 90.9\end{array}$ & $\begin{array}{l}84.1 \\
89.8\end{array}$ & $\begin{array}{l}74.7 \text { to } 91.0 \\
84.8 \text { to } 93.5\end{array}$ \\
\hline \\
\hline $\begin{array}{l}\text { Short term } \\
\text { Long term }\end{array}$ & $\begin{array}{l}84.3 \\
59.4\end{array}$ & $\begin{array}{l}75.8 \text { to } 90.8 \\
40.0 \text { to } 76.3\end{array}$ & $\begin{array}{l}80.6 \\
90.2\end{array}$ & $\begin{array}{l}68.6 \text { to } 89.6 \\
83.9 \text { to } 94.7\end{array}$ \\
\hline & & & & \\
\hline $\begin{array}{l}\text { Short time } \\
\text { Long term }\end{array}$ & $\begin{array}{l}88.8 \\
68.2\end{array}$ & $\begin{array}{l}79.7 \text { to } 94.7 \\
45.1 \text { to } 86.2\end{array}$ & $\begin{array}{l}72.4 \\
87.8\end{array}$ & $\begin{array}{l}60.9 \text { to } 82.0 \\
80.9 \text { to } 92.9\end{array}$ \\
\hline
\end{tabular}

$\star$ Sensitivity: at least one disability among those limited in activities. †pecificity: no disabilities among those not limited in activities. $\mathrm{CI}=$ confidence intervals.
Table 4 Prevalence of disabilities * by ISS

\begin{tabular}{|c|c|c|c|c|c|}
\hline \multirow[b]{2}{*}{ ISS } & \multirow[b]{2}{*}{ No } & \multicolumn{2}{|c|}{ Short term } & \multicolumn{2}{|c|}{ Long term } \\
\hline & & $\%$ & $95 \% C I$ & $\%$ & $95 \% C I$ \\
\hline $\begin{array}{l}1-2 \\
4-8 \\
9-41\end{array}$ & $\begin{array}{l}53 \\
88 \\
49\end{array}$ & $\begin{array}{l}35.8 \\
63.6 \\
83.7\end{array}$ & $\begin{array}{l}23.1 \text { to } 50.2 \\
52.7 \text { to } 73.6 \\
70.3 \text { to } 92.7\end{array}$ & $\begin{array}{r}3.8 \\
23.3 \\
20.4\end{array}$ & $\begin{array}{r}0.4 \text { to } 13.0 \\
14.8 \text { to } 33.6 \\
10.2 \text { to } 34.3\end{array}$ \\
\hline
\end{tabular}

*At least one disability in the disability scale.

$\mathrm{CI}=$ confidence interval.

associated with injury severity as measured by the ISS (table 4). A majority of children with injuries with a ISS 9 or above experience 5 short term disabilities, while about one in fivechildren with an ISS of 4 and over stillw experienced disabilities six months after the injury.

\section{Discussion}

Data sets have been developed in differen? countries for the study of injuries requiring emergency department services. $^{2122}$ In the WHO surveillance form, questions about limitations in activities are integrated, ${ }^{23}$ ande the International Collaborative Effort on Injury Statistics discussed the inclusion of outcomes as part of a basic data set. ${ }^{24}$

The seven item scale presented here is simple instrument that could be integrated into̊ such a data set. The scale is easy to use and? score, and it can be applied by lay interviewer $\vec{D}$ after short training. It takes a short time to complete, estimated at around one minute Although it has few items, they are compre hensive. The questions are simple, covering mainly motor activities and those of persona care. They reflect activities of the child irrespective of the context in which he/she performs them, and measures disabilities irre spective of the underlying injury. The type of items studied seem not to be affected by development, and hence the scale is applicable to children and adolescents.

Answers are based on the parent's appraisa? of the child's performance of activities. The 'yes/no' type of response seems suitable be cause it has been proposed ${ }^{8}$ that parents have. difficulty ascertaining the qualitative aspects of disability. The response of parents was elicited for all children and adolescents to assure uniformity in the answers. Parents were usually the proxy respondents for other members of the family in surveys on disabilities in different countries. ${ }^{25}$ The likelihood that there will be misclassification of the child's capabilities due to a biased ascertainment by the parents canno $\overline{0}$ be elucidated and we don't know whether this will cause over or under-reporting of the disabilities in the different age groups. The use of usual activities as the gold standard can be considered acceptable, because no othef appropriate measure has yet been defined for that purpose. ${ }^{8}$ The fact that the scale and goles standard questions were asked in the same interview may be a potential source of bias. It is difficult to state, however, which is the best time interval that should be allowed for the testing of validity. 
Internal consistency-reliability was relatively high mainly for the short term disabilities and persisted across categories of the sociodemographic variables. Thus, the scale is suitable for populations of different educational and sociocultural backgrounds. It compares favourably with the generic 10 item scale tested by Zaman et al to study disabilities among 2-9 year olds in a general population. ${ }^{26}$

The scale also has face validity, because it includes basic and personal care activities performed by children in most cultures. The correlation with the comprehensive 25 item scale $^{16}$ was high, and a significant association was found with the ISS, a score that measures the severity of the injury but not impairments or disabilities resulting from it. ${ }^{18}$ Construct validity is acceptable, mainly for the short term disabilities. Sensitivity and specificity were lower than the 10 item scale, ${ }^{26}$ where a professional's diagnosis served as the gold standard. The decrease in the prevalence of disabilities six months after the injury, and the inclusion of disabilities regardless of their severity, affected the sensitivity of the long term scale. The scale was categorized as 0 and $1+$ throughout the study. It might be of value to look at a more detailed categorization in a larger population, because the present one was too small for such an analysis.

In conclusion, the seven item disability scale could be used to assess disabilities after injury among children and adolescents in different cultures. It involves a simple method, that does not require expert personnel. Its use may be more effective to detect disabilities shortly after the injury, or to determine period prevalence of disabilities, than for those disabilities persisting after six months. For the purpose of injury prevention, this scale can be used in addition to measures of frequency and severity to define high risk groups and priorities for intervention.

We are grateful to Tamar Hass MPH, for her contribution during data collection.

The study was partially funded by the Joint Research Fund of the Hebrew University and Hadassah.

1 Rivara FP, Calogne N, Thompson RS. Population-based study of unintentional injury incidence and impact during childhood. Am ₹ Public Health 1989; 79: 990-4.

2 Barker M, Power C, Roberts I. Injuries and the risk of disability in teenagers and young adults. Arch Dis Child disability in teenagers

3 Collins JG. Persons injured and disability days due to injuries. United States, 1980-81. Hyattsville, MD: US Department of Health and Human Services. Public Health Service.
National Center for Health Statistics. Series 10, No 149, 1985

4 World Health Organization. International classification of impairments, disabilities and handicaps. Geneva: WHO, 1980.

5 Msall ME, DiGaudio K, Duffy LC, LaForest S, Braun S, Granger CV. WeeFIM. Normative sample of an instrument for tracking functional independence in children. ment for tracking functional independe

6 Eisen M, Donald CA, Ware JE, Brook RH. Conceptualization and measurement of health for children in the health insurance study. Santa Monica, CA: Rand Corporation, 1985.

7 Thorburn MJ, Desai P, Durkin M. A comparison of efficacy of the key informant and community survey methods in the identification of childhood disability in Jamaica. $A n n$ Epidemiol 1991; 1: 255-61.

8 Young LY, Yoshida K, Williams JI, Bombardier C, Wright JG. The role of children in reporting their physical disability. Arch Phys Med Rehabil 1995; 76: 913-8.

9 Yates DW, Heath DF, Mars E, Taylor RJ. A system for measuring the severity of temporary and permanent
disability after injury. Accid Anal Prev 1991; 23: 323-9.

10 States JD, Viano DC. Injury impairment and disability scales to assess the permanent consequences of trauma. Accid to assess the permanent conseq

11 Haley SM, Coster WJ, Ludlow LH, Haltiwanger J, Andrellos P. Pediatric evaluation of disability inventory (PEDI). Version I: development, standardization and administration manual. Boston, MA: New England Medical Center-PEDI Research Group, 1992.

12 Jennett B, Teasdale G, Braakman R, et al. Prognosis of patients with severe head injury. Neurosurgery 1979; 4 283-9.

13 Furrie AD. The Canadian data base on disability issues: a national application of the ICIDH. Disabil Rehabil 1995; 17: $344-9$.

14 Durkin MS, Wang W, Shrout PE, et al. Evaluating a ten questions screen for childhood disability: reliability and internal structure in different cultures. $\mathscr{f}$ Clin Epidemio 1995; 48: 657-66.

15 Langley JD, Stanton WR, McGee RO, Murdoch JC. Disability in late adolescence $I$ : introduction, methods and overview. Disabil Rehabil 1995; 17: 35-42.

16 Gofin R, Hass T, Adler B. The development of disability scales for childhood and adolescent injuries. $₹ \mathrm{Clin}$ Epidemiol 1995; 48: 977-84.

17 World Health Organization. International classification of diseases. 9th Revision (ICD-9-CM). Geneva: WHO, 1978.

18 Association for the Advancement of Medicine. The abbreviated injury scale. 1990 Revision. Des Plaines, II: AAM,

19 SPSS Inc. SPSS/PC+V2.0 base manual. Chicago, IL: SPSS Inc, 1988.

20 Gallingher PM, Abramson JH. Computer program for epidemiological analysis-PEPI version 2. Stone Mountain, GA: USD Inc, 1995.

21 National Injury Surveillance Unit. National minimum datase for injury surveillance. Australian Institute of Health and Welfare, Version 1.1, 1994

22 Lund J. Integrated data collection systems at hospitals. Proceedings of the International Seminar on Accident Data. Rogman W, Schurman M, eds. Baden (near Vienna) 13Rogman W, Sch April, 1989.

23 World Health Organization. Prevention of accidents. $A$ basic data set and guidelines for its use. (EUR/CP/APR113 0657j.) Geneva: WHO, 1988

24 Minimum basic data set (MBDS), unintentional injuries. Proceedings of the International Collaborative Effort on Injury Statistics. Volume I. US Department of Health and Human Services. Public Health Service. Centers for Disease Control and Prevention. National Center for Health Statistics. DHHS Publication No (PHS) 95-1252. Hyattsville, MD, 1995: 34-1-34-4.

25 Gorter KA. Survey methods for the assessment of physical disability among children. Disabil Rehabil 1993; 15: $47-$ 51.

26 Zaman SS, Khan NZ, Islam S, et al. Validity of the 'ten questions' for screening serious childhood disability: results from urban Bangladesh. Int $\mathcal{F}$ Epidemiol 1990; 19: $613-20$

\section{Birthday boy dies jumping for joy}

A boy who liked to use his bunk bed as a trampoline broke his neck when he fell off and crashed into a safety net made by his parents. The boy was found dead by his mother on the day that the family was to hold his 11 th birthday party.

His bed stood 4'2" off the ground and was made from scaffolding poles because he had broken three previous beds using them as trampolines. His father, an electronics research engineer from Northumberland, said: ' $\mathrm{He}$ was so excited about his party. He was such a livewire, always rushing around. Everyone who knew him would tell how full of energy he was. It is terrible that he died like this. It looks as though he had been trying to do back somersaults on his bed and something went horribly wrong. He used to enjoy climbing about in his bedroom, making dens and caves around the furniture' (The Times, November 1996). 